\title{
Intestinal histoplasmosis in immunocompetent patient: An unusual cause of massive small intestine bleeding
}

\author{
Elisa Jiménez-Rivera ${ }^{1 *}$, Ediel O. Dávila-Ruiz¹, Roberto A. García-Manzano ${ }^{1}$, Gustavo A. Santos-Chab, \\ José M. Aguirre-Trejo ${ }^{1}$, Javier Fabián Fabián ${ }^{1}$, and Fabián Tafoya-Ramirez ${ }^{2}$ \\ ${ }^{1}$ Departament of General Surgery and Minimal Invasion; ${ }^{2}$ Departament of de Anatomy Pathology. Hospital Regional de Alta Especialidad de Oaxaca, \\ San Bartolo Coyotepec, Oaxaca, Mexico
}

\begin{abstract}
Small bowel bleeding (SBB) accounts for $5-10 \%$ of patients with gastrointestinal bleeding and its association is unusual in patients with histoplasmosis. The incidence of gastrointestinal histoplasmosis is unknown since only 3-12\% present symptoms, predominantly in immunocompromised patients. However, in immunocompetent hosts, it is reported only in $0.05 \%$ of cases. A clinical case of an immunocompetent patient with massive SBB, Grade IV hypovolemic shock associated with systemic histoplasmosis and treated with intestinal resection and amphotericin $B$ is hereby discussed.
\end{abstract}

Key words: Intestinal histoplasmosis. Disseminated histoplasmosis. Small bowel bleeding.

\section{Introduction}

Small bowel bleeding (SBB) is a rare entity, accounting for $\sim 5-10 \%$ of all gastrointestinal bleeding cases ${ }^{1}$. Causes of SBB in $<40$ years are inflammatory bowel disease, Dieulafoy's lesion, neoplasms, Meckel's diverticulum, and polyps. Gastrointestinal tract bleeding in patients with disseminated histoplasmosis and gastrointestinal involvement is unusual. The exact incidence of gastrointestinal histoplasmosis $(\mathrm{GIH})$ is unknown because most patients remain asymptomatic ${ }^{2}$, only $3-12 \%$ of patients show symptoms ${ }^{3}$, it is reported in $70 \%$ of autopsies in immunosuppressed patients (HIV and transplant recipients) ${ }^{2}$ and in immunocompetent hosts in only $0.05 \%$ of cases $^{4}$. Histoplasmosis is also known as Darling's disease (1906), cave fever, or abandoned mine fever ${ }^{5}$. The etiological agent is the dimorphic fungus Histoplasma capsulatum var. capsulatum, the mycelial or saprophytic infecting form, found in bat or bird excreta $^{6}$. It is considered the most frequent deep mycosis in North America and the second most frequent in South America ${ }^{5}$, with microfoci in East United States, South Europe, Africa, and Southeast Asia ${ }^{7}$. In Mexico, it is the most important systemic mycosis, according to the reports of outbreaks during 1988-1994, it is endemic throughout the country, predominantly in Morelos, Guerrero, Veracruz ${ }^{8}$, and Oaxaca ${ }^{6}$. We present a clinical case of an immunocompetent patient with massive SBB and Grade IV hypovolemic shock associated with systemic histoplasmosis, treated with intestinal resection and amphotericin B.

\section{Clinical case}

A 29-year-old male patient, native and resident of Oaxaca, farmer and rubbish collector, with a history of alcohol and methamphetamine abuse, starts clinical condition of 6 months with colicky abdominal pain in

\section{Correspondence:}

*Elisa Jiménez-Rivera

E-mail: eli_jr3@ hotmail.com
Available online: $29-07-2021$ Rev Med Hosp Gen Mex. 2021;84(3):123-128 www.hospitalgeneral.mx 0185-1063/@ 2020 Sociedad Médica del Hospital General de Mexico. Published by Permanyer. This is an open access article under the CC BY-NC-ND license (http://creativecommons.org/licenses/by-nc-nd/4.0/). 
hypogastrium, intensity $7 / 10$ in VAS, evening fever, weight loss of $20 \mathrm{~kg}$ in 2 months, diarrhea and constipation, choluria, and non-productive cough. He was admitted to the emergency department for abdominal pain, respiratory distress, altered state of consciousness and hemodynamic instability, heart rate $120 \mathrm{bpm}$, respiratory rate $22 \mathrm{rpm}$, mean arterial pressure $60 \mathrm{mmHg}$, temperature $38.9^{\circ} \mathrm{C}$, rales in the left lower lobe, painful abdomen in the left iliac fossa, and hepatomegaly. Laboratory studies were requested, reporting Grade I anemia, hypoalbuminemia, moderate hyponatremia, prolonged coagulation times, blood chemistry and viral panel without alterations, and discrete elevation of fibrinogen (Table 1). Computed tomography (CT) scans of the chest and abdomen (Figs. 1-2) with images suggestive of multisegmental pneumonia, hepatomegaly, mesenteric and retroperitoneal lymphadenopathy, ascites, concentric thickening $12 \mathrm{~mm}$ of the wall of the descending colon and sigmoid colon, and endoscopy without alterations. Colonoscopy reported erosions in the mucosa at the proximal sigmoid level, without passage to the distal ileum due to ileocecal valve stenosis. A biopsy was taken that reported moderate chronic erosive colitis, not active. Four days later, he presented with acute abdomen accompanied by hematemesis and hematochezia leading to hypovolemic shock Grade IV, hemoglobin $6.1 \mathrm{mg} / \mathrm{dl}$, platelets $52,000 \mathrm{mg} / \mathrm{dl}$, TP $29.1 \mathrm{~s}$, thrombotic thrombocytopenic purpura $49 \mathrm{~s}$, and INR $2.2 \mathrm{~s}$. The patient underwent emergency surgery and was found to have an ileum-dependent tumor measuring $8 \times 5 \times 4 \mathrm{~cm}$ (Fig. 3), $1 \mathrm{~m}$ from the ileocecal valve. It involved the parietal peritoneum and sigmoid colon, with abundant lymph nodes in the root of the mesentery larger than $1 \mathrm{~cm}$ in diameter. The tumor was resected en bloc including $60 \mathrm{~cm}$ of terminal ileum and $30 \mathrm{~cm}$ of sigmoid colon with a $60 \mathrm{~mm}$ GIA linear stapler, ileostomy of the proximal end, closure of the distal end of the remaining $70 \mathrm{~cm}$ ileum and end-to-end anastomosis of the descending colon, and the remaining sigmoid colon. The histopathological report of the surgical specimen showed chronic inflammation, deep ulceration of the mucosa with abundant intracellular microorganisms in yeast-like macrophages positive for GROCOTT, and periodic acid-Schiff staining (Figs. 4-5). The diagnosis of intestinal Histoplasmosis was established and treatment was started with liposomal Amphotericin B at a dose of $5 \mathrm{mg} / \mathrm{kg} /$ day. One week after treatment, he presented cardiac arrhythmia and sudden death.
Table 1. Values reported in laboratory studies on patient admission

\begin{tabular}{|l|c|}
\hline $\begin{array}{l}\text { Blood biometry } \\
\text { Leukocytes }\end{array}$ & $9.3 \times 103 / \mathrm{mm}^{3}$ \\
Neutrophils & $7.3 \times 103 / \mathrm{mm}^{3}$ \\
Hemoglobin & $10 \mathrm{~g} / \mathrm{dl}$ \\
Platelets & $221 \times 105 / \mathrm{mm}^{3}$ \\
\hline Blood chemistry & \\
Glucose & $81 \mathrm{mg} / \mathrm{dl}$ \\
\hline Urea & $8.5 \mathrm{mg} / \mathrm{dl}$ \\
BUN & $1.2 \mathrm{mg} / \mathrm{dl}$ \\
Creatinine & $0.5 \mathrm{mg} / \mathrm{dl}$ \\
Uric acid & $1.2 \mathrm{mg} / \mathrm{dl}$ \\
\hline Coagulation times & \\
TP & $21.2 \mathrm{seg}$ \\
TTPa & $84 \mathrm{seg}$ \\
INR & $1.5 \mathrm{seg}$ \\
Fibrinogen & $448 \mathrm{mg} / \mathrm{dl}$ \\
\hline Special tests & \\
HIV-1 and HIV-2 & Non-reactive \\
TORCH profile & Non-reactive \\
Guayaco & Positive \\
\hline Tumor markers & \\
Ca 19-9 & $186.4 \mathrm{UI} / \mathrm{L}$ \\
CAE & $1.58 \mathrm{ng} / \mathrm{ml}$ \\
Alpha-fetoprotein & $1.94 \mathrm{ng} / \mathrm{dl}$. \\
\hline
\end{tabular}

\section{Discussion}

Histoplasma infection starts with inhalation of aerosolized microspores of microconidia and hyphae ${ }^{6}$, in the alveolar sac, they are phagocytosed by macrophages, the conidia develop into yeast ${ }^{5}$ at $35-37^{\circ} \mathrm{C}^{4}$ and reach the macrophage interior to multiply ${ }^{5}$ until the T-cell response. Primary Histoplasma infection is asymptomatic and remains dormant ${ }^{4},<5 \%$ of exposed individuals develop symptoms. Cell-mediated immunity favors hematogenous spread of the fungus especially to the reticuloendothelial system ${ }^{3}$. The immune response blocks its progression by forming calcified granulomas in the affected organs ${ }^{5}$, and if the T-cell response is inadequate, it reactivates ${ }^{4}$. The most susceptible are patients with AIDS, HTLV-1 infection, hepatitis $C$ infection, renal failure, glucocorticosteroid use and biological agents ${ }^{9}$, as well as methamphetamine $u^{10} e^{10}$. Disseminated disease in immunocompetent patients is found in $<0.05 \%{ }^{4}$ and only nine cases of GIH in immunocompetent patients have been reported ${ }^{2}$, probably due to an unknown defect in cell-mediated immunity ${ }^{4}$. Disseminated histoplasmosis is rare and lesions in the gastrointestinal tract are infrequent, manifesting as esophageal involvement in mediastinal adenitis, mediastinal fibrosis 


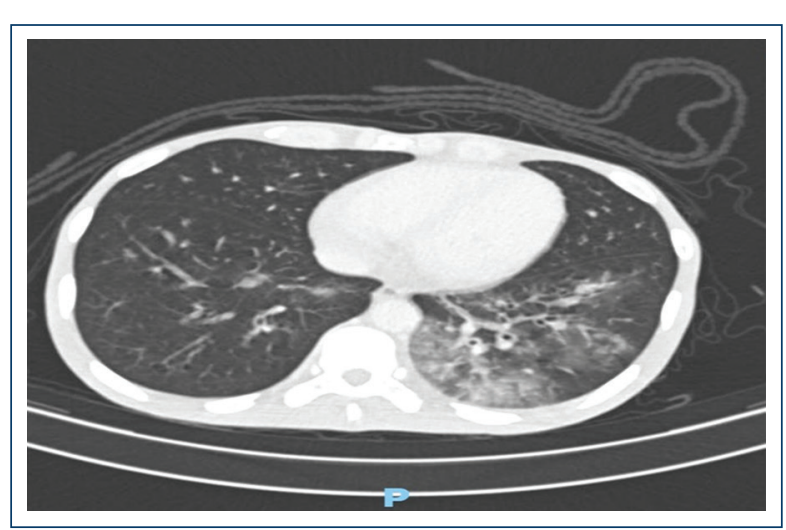

Figure 1. Plain and contrasted tomography of the thorax with evidence of ground-glass opacity, an image suggestive of the left multisegmental pneumonia.
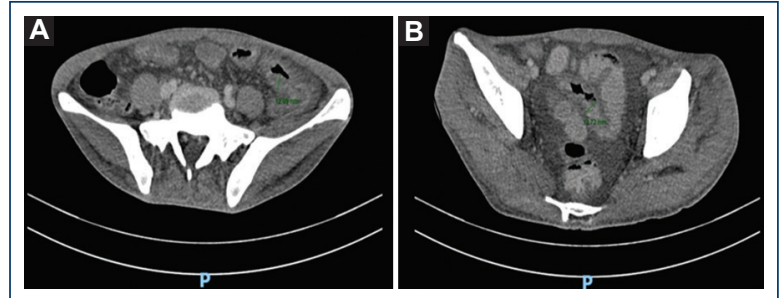

Figure 2. A: simple and contrasted tomography of the abdomen is evident in the descending colon and sigmoid colon. B: thickening of the colon wall with a maximum diameter of $12 \mathrm{~mm}$.

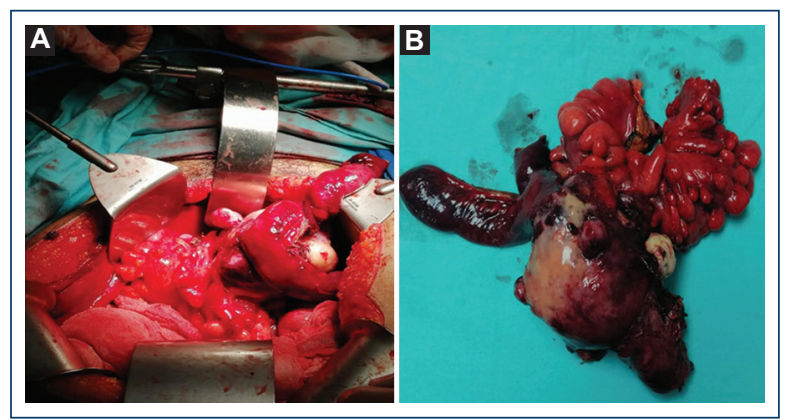

Figure 3. A: $8 \times 5 \times 4 \mathrm{~cm}$ tumor involving parietal peritoneum at the level of the left deep inguinal ring dependent on the distal ileum. B: surgical specimen of ileum-dependent tumor, which was resected with a margin of $10 \mathrm{~cm}$ of lesion.

with esophageal involvement, and disseminated histoplasmosis with gastrointestinal involvement. The latter is frequent in men in the fifth decade of life ${ }^{4}$ and can occur at any site of the gastrointestinal tract ${ }^{9}$, the

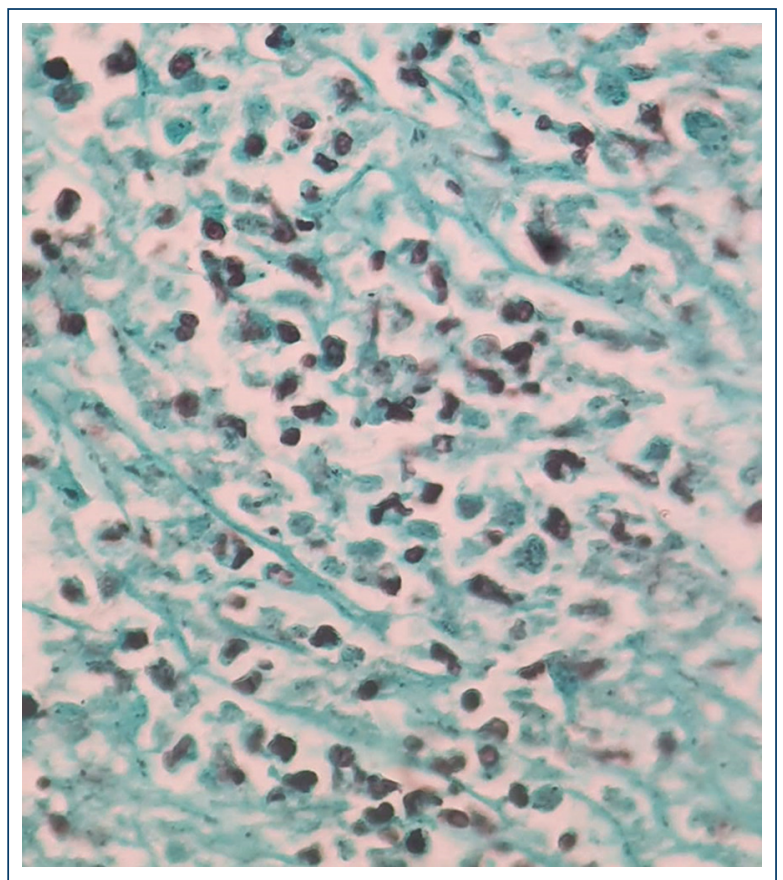

Figure 4. Grocott stain showing spherical, pyriform, nailshaped microconidia, thin-walled with no septa.

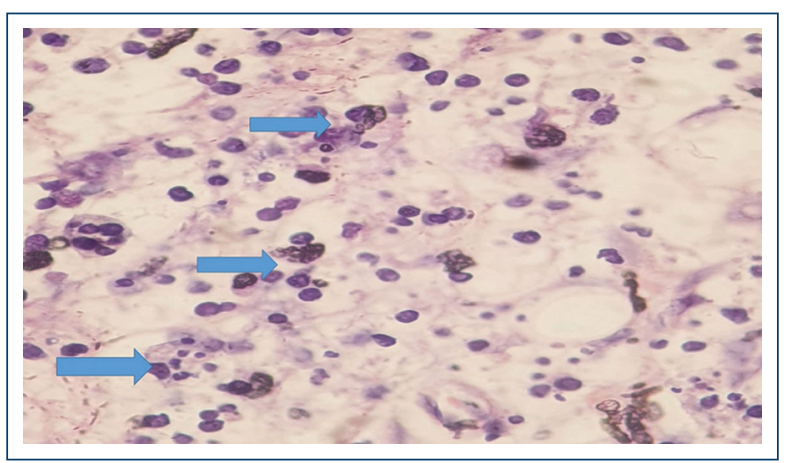

Figure 5. Periodic acid-Schiff stained sample, small isolated round to oval yeast-like rosette structures forming clusters, corresponding to Histoplasma spp. (blue arrow).

terminal ileum being the most common site because of its rich lymphatic system, manifesting with ulcers ${ }^{11}$, as in the reported case. About $30-50 \%$ of patients with GIH report non-specific abdominal pain, fever, weight loss or diarrhoea ${ }^{2}$, ulcers, polyps, strictures, and perforations may be present ${ }^{5}$, complications are rare such as bleeding and protein loss due to enteropathy and hypogammaglobulinaemia ${ }^{9}$. Bleeding is more common in AIDS patients than in immunocompetent patients $(18 \% \text { vs. } 9 \%)^{3}$, and three renal transplant patients with intestinal perforation have been 
reported ${ }^{12}$, patients with hemorrhage present hematochezia or melena, depending on site and intensity ${ }^{13}$. Immunosuppressed patients may begin with obstruction by annular, constricting lesions, or large polypoid masses, from the rectum to the cecum, resembling appendicitis, malignant neoplasia, or inflammatory bowel disease ${ }^{3}$. The clinical case reported is a male patient in his third decade of life, a farmer and rubbish collector, which predisposes him to contact with the infective forms of Histoplasma. Although no apparent cause of immunocompromise was documented, the patient had risk factors that predisposed him to a decrease in the immune response, the consumption of methamphetamines, according to Potula et al., demonstrated that stimulants such as methamphetamine (METH) exert immunosuppressive effects on the host innate and adaptive immune systems, because METH can limit T-cell proliferation by exerting a prolonged $\mathrm{G} 1 / \mathrm{S}$ phase in the cell cycle and a significant decrease in the expression of cyclin E, CDK2, and transcription factor $\mathrm{E} 2 \mathrm{~F} 1^{10}$. Similarly, hypoalbuminemia and alcohol consumption correlate with poor nutritional status of the patient. In a retrospective study of pediatric patients diagnosed with histoplasmosis, malnutrition was identified as the most important risk factor because it is considered the leading cause of secondary immunodeficiency in the world, decreasing the host immune response, including cell-mediated immunity ${ }^{14}$. The diagnosis of $\mathrm{GlH}$, either by exclusion or as a finding, its differential diagnosis includes chronic inflammatory processes such as Crohn's disease, cancer, and intestinal tuberculosis. Laboratory findings may be non-specific, although they reflect signs of bone marrow involvement (anemia, leukopenia, and plateletopenia) and liver function $^{5}$. In cases of suspected histoplasmosis, direct microscopic examination, culture, antigen detection, and serological tests for antibodies are used for diagnosis ${ }^{3}$. Multiphasic CT or CTA can detect bleeding of $0.3 \mathrm{ml} / \mathrm{min}$; however, the patient must be actively bleeding at the time of the study, findings of blood within the lumen or sentinel clot help localize subtle or absent bleeding ${ }^{1}$. GIH images are bowel wall thickening, large masses, signs of small bowel obstruction, and free peritoneal air, while findings of disseminated histoplasmosis are hepatosplenomegaly, pulmonary infiltrates, and generalized intra-abdominal lymphadenopathy ${ }^{3}$. In the clinical case, the patient began with non-specific gastrointestinal symptoms and pneumonia. The initial CTA did not identify an active bleeding site. However, the data found were multisegmental pneumonia, hepatomegaly, mesenteric adenopathies, and thickening of the wall of the descending colon. The gastrointestinal tract bleeding presented as fecal occult blood, which progressed to Grade IV hypovolemic shock. Prolonged coagulation times and decreased platelet levels were the factors associated with bleeding, at the expense of a slight elevation of fibrinogen on admission, probably because the average half-life of fibrinogen is $100 \mathrm{~h}$, the patient presented bleeding data until the $4^{\text {th }}$ day of inpatient stay. Direct visualization and biopsy for culture and pathology are essential for the diagnosis of both gastrointestinal tract bleeding and intestinal histoplasmosis. The algorithm established by the American College of Gastroenterology divides SBB into patients with stable or unstable hemodynamic status; the former are candidates for endoscopy, and if this is not satisfactory, an auxiliary method is used; in unstable patients, resuscitation measures are initiated, followed by imaging, endoscopic, surgical, or combined studies to demonstrate the site of bleeding 1 . If no bleeding site is found in the first endoscopy, capsule endoscopy is used to identify SBB, an unrecognized lesion in the stomach or colon ${ }^{13}$. However, few centers in Spain have this tool, so in the event of a second endoscopic examination, push enteroscopy is an ideal procedure; another tool is intraoperative enteroscopy (IOE), which involves the evaluation of the small intestine during laparotomy ${ }^{1}$. Lesions found in GIH include single or continuous superficial mucosal ulcers, deep bleeding ulcers with or without frank perforations, areas of friable and mass containing necrosis, and obstructions due to circumferential exophytic thickening ${ }^{9}$. Diffuse ulceration was observed in $85.7 \%$ of cases with AIDS-related $\mathrm{GIH}^{4}$. In the patient reported in the clinical case, colonoscopy identified erosions at the level of the proximal sigma that stands out on surface staining and non-active moderate chronic erosive colitis. Transoperative findings included ileum-dependent tumor leading to significant bleeding and transoperative endoscopic findings of fibrin-covered lesions and areas of active subepithelial hemorrhage up to $20 \mathrm{~cm}$ of ileum. In the histopathological report of $\mathrm{GIH}$, macrophages in the mucosa and submucosa show round eosinophilic organisms, 1-4 $\mu \mathrm{m}$ in size with a transparent halo around them ${ }^{4}$. Four different pathological forms have been recognised ${ }^{9}$ (Table 2). In the documented case, the pathological form presented was type IV due to localized thickening at the level of the distal ileum involving the sigmoid segment. 
Table 2. Pathological forms of gastrointestinal histoplasmosis

I Subclinical, microscopic clusters of macrophages in the lamina propria (LP)

II Plaques and pseudopolyps caused by fungi contained in macrophages

III Tissue necrosis and ulceration leading to abdominal pain, diarrhea, and bleeding

IV Localized thickening with inflammation of the bowel, simulates cancer, or Crohn's disease

Histoplasmosis can be diagnosed by serum tests, complement fixation, radioimmunoassay, precipitation test, polymerase chain reaction, and microscopy. Grocott stained culture is the gold standard for diagnosis as it stains the capsule around the yeast forms ${ }^{4}$. However, it is not recommended as an initial study because it takes 4-6 weeks for the organism to grow 4 . The reported patient's diagnosis was histopathological by Grocott staining 10 days after the surgical event.

Treatment of mild-to-moderate GIH is itraconazole $200 \mathrm{mg} 3$ times daily for 3 days and then twice daily for at least 12 months $^{15}$. In severe cases, liposomal amphotericin B $3.0 \mathrm{mg} / \mathrm{kg}$ daily for $1-2$ weeks, followed by oral itraconazole $200 \mathrm{mg} 3$ times daily for 3 days and continue $200 \mathrm{mg}$ twice daily for at least 12 months. Treatment of SBB should be managed according to the algorithms for STD, massive bleeding associated with histoplasmosis has only been reported by Bruno et al., in a patient in association with cytomegalovirus and AIDS, finding a $2 \times 2 \mathrm{~cm}$ tumor in the distal ileum ${ }^{16}$. Surgical treatment for SBB is usually considered as a last resort and is guided by IOE, whenever possible. Indications for emergency surgery include hemodynamic instability with active bleeding, persistent recurrent bleeding, or the need for transfusion of more than 6 units of red blood cell concentrate in $24 \mathrm{~h}$ with active bleeding ${ }^{17}$. When the location of the hemorrhage is known, segmental resection ensures recovery with a mortality rate of $<3-5 \%$ and a recurrence rate of $2-10 \%{ }^{16}$.

\section{Conclusion}

Gastrointestinal tract bleeding of unknown origin associated with GIH is a very rare entity in immunocompetent patients, with nutritional status and methamphetamine use being predisposing risk factors for its activation. Due to its characteristics, a diagnosis of exclusion makes it necessary to rule out pathologies such as Crohn's disease, UC, lymphoproliferative processes, and intestinal tuberculosis. Appropriate treatment leads to long-term survival, while untreated cases are almost fatal, especially when there is an association with gastrointestinal tract bleeding. Hence, joint treatment should be carried out and in the event of massive bleeding secondary to $\mathrm{GlH}$, surgical resection should be considered if the site of bleeding is identified.

\section{Acknowledgments}

Thanks to all contributors to this article who were directly and indirectly involved, as well as for their support, encouragement, and incentive.

\section{Conflicts of interest}

The authors declare that there are no conflicts of interest in this article.

\section{Funding}

The authors declare that no funding was involved in the performance of this study.

\section{Ethical disclosures}

Protection of human and animal subjects. The authors declare that no experiments were performed on humans or animals for this study.

Confidentiality of data. The authors declare that they have followed the protocols of their work center on the publication of patient data.

Right to privacy and informed consent. The authors have obtained the written informed consent of the patients or subjects mentioned in the article. The corresponding author is in possession of this document.

\section{References}

1. Gerson LB, Fidler JL, Cave DR, Leighton JA. ACG clinical guideline: diagnosis and management of small bowel bleeding. Am J Gastroenterol. 2015;110:1265-88.

2. Dang $Y$, Jiang L, Zhang J, Pan B, Zhu G, Zhu F, et al. Disseminated histoplasmosis in an immunocompetent individual diagnosed with gastrointestinal endoscopy: a case report. BMC Infect Dis. 2019;19:992.

3. Kahi CJ, Wheat LJ, Allen SD, Sarosi GA. Gastrointestinal histoplasmosis. Am J Gastroenterol. 2005;100:220-31.

4. Sharma R, Lipi L, Gajendra S, Mohapatra I, Goel RK, Duggal R, et al. Gastrointestinal histoplasmosis: a case series. Int J Surg Pathol. 2017;25:592-8.

5. Montero A. Histoplasmosis. In: Medicina Tropical. $1^{\text {st }}$ ed. Barcelona: Elsevier; 2013. p. 257-74, 304. 
6. Contreras $\mathrm{C}$, Shibayama H, Gutiérrez P. Aportaciones del instituto nacional de diagnóstico y referencia epidemiológica a la histoplasmosis. Rev Inst Nal Enf Respir Mex. 1998;11:216-20.

7. Gutiérrez-Casillas S, Flores-Rivera OI, Alonso-Martínez D, Macías RF, Espino-López E. Enfermedad del viajero. Histoplasmosis diseminada en paciente inmunocompetente. Med Int Méx 2014;30:335-8.

8. Corcho-Berdugo A, Muñoz-HernándezB,Palma-Cortés G, Ramírez-HernándezA Martínez-Rivera M, Frías-de León M, et al. Brote inusual de histoplasmosis en residentes del estado de México. Gaceta Méd Méx. 2011;147:377-84.

9. Zhu LL, Wang J, Wang ZJ, Wang YP, Yang JL. Intestinal histoplasmosis in immunocompetent adults. World J Gastroenterol. 2016;22:4027-33.

10. Potula R, Haldar B, Cenna JM, Sriram U, Fan S. Methamphetamine alters $T$ cell cycle entry and progression: role in immune dysfunction. Cell Death Discov. 2018;4:44.

11. Ai XB, Wang ZJ, Dong QC, Lin X, Chen YP, Gong FY, et al. Ileum histoplasmosis mimicking intestinal tuberculosis and Crohn's disease. Case Rep Gastroenterol. 2018;12:63-8.
12. Syed TA, Salem G, Kastens DJ. Lower gastrointestinal bleeding secondary to intestinal histoplasmosis in a renal transplant patient. ACG Case Rep J. 2017;4:e93.

13. Kuo JR, Pasha SF, Leighton JA. The Clinician's guide to suspected small bowel bleeding. Am J Gastroenterol. 2019;114:591-8.

14. López LF, Valencia Y, Tobón ÁM, Velásquez O, Santa CD, Cáceres DH, et al. Childhood histoplasmosis in Colombia: clinical and laboratory observations of 45 patients. Med Mycol. 2016;54:677-83.

15. Wheat LJ, Freifeld AG, Kleiman MB, Baddley JW, McKinsey DS, Loyd JE, et al. Clinical practice guidelines for the management of patients with histoplasmosis: infectious diseases society of America. Clin Infect Dis. 2007;45:807-25

16. Bruno MA, Parodi M, Olmedo I, Molina HP, Palencia R, Doniquian A. Massive gastrointestinal bleeding in AIDS patients secondary to histoplasma and cytomegalovirus infection. Int J Surg Case Rep. 2016;25:128-31.

17. Feinman M, Haut ER. Lower gastrointestinal bleeding. Surg Clin North Am. 2014:94:55-63. 